\title{
Rehabilitation Center
}

National Cancer Institute

\section{Source}

National Cancer Institute. Rehabilitation Center. NCI Thesaurus. Code C53535.

A healthcare facility designed for improving, maintaining, or restoring physical strength, cognition, or mobility. 